\title{
Advanced Development of Carboxylic Acid Functionalized Multiwall Carbon Nanotubes as Safe Inhalation Drug Carrier
}

\author{
Krishnat Dhekale, Sharvil Patil, Ravindra Kamble* \\ Department of Pharmaceutics, Poona College of Pharmacy, Bharati Vidyapeeth University, Erandwane, Pune, Maharashtra, INDIA.
}

\begin{abstract}
Background: Multiwall Carbon Nanotubes (MWCNTs) have innovative characteristic features strongly associated with nanotechnology and drug delivery applications. Objectives: The current work was performed with an objective to assess the inhalation toxicity of multi-walled carbon nanotubes. Methods: Functionalization of multi-walled carbon nanotubes was carried out by the initial basic treatment with hydrochloric acid and nearly assessed by Infrared (IR) spectroscopy, mass spectroscopy, and Transmission electron microscopy (TEM) in comparison with pure multi-walled carbon nanotubes. The acute inhalation toxicity of these functionalized and pure multi-walled carbon nanotubes was assessed in Wistar rats. The parameters: hematology, liver function test, kidney function test, and histopathology observed, evaluated and interpreted for the toxicity study. Results: The functionalized multi-walled carbon nanotubes and pure multi-walled carbon nanotubes were nearly assessed by IR spectroscopy, mass spectroscopy, and TEM. The assessments affirmed and anticipated that the pure multi-walled carbon nanotube undergoes successful functionalization. Results obtained showed a remarkable difference in the ranges of hemoglobin and platelet. The acute inhalation toxicity study did not show any toxicity of functionalized multi-walled carbon nanotubes
\end{abstract}

when compared to pure multi-walled carbon nanotubes. The results of acute inhalation toxicity studies did not show any significant changes also not demonstrated hazard potential in rats following acute exposures to the functionalized multi-walled carbon nanotubes and there is no significant toxicity. Because of basic treatment during functionalization all impurity gets removed. During functionalization addition of oxygen containing group and removal of carbon dioxide group occurs. Conclusion: Functionalized multi-walled carbon nanotubes can prove to be a novel, safe, and non-toxic carrier for the delivery of drugs by inhalation route.

Key words: Carbon nanotubes, Functionalization, Acute inhalation toxicity, Dry powder inhaler, Liver function test, Kidney function test.

\section{Correspondence}

Dr. Ravindra Kamble

Department of Pharmaceutics, Poona College of Pharmacy, Bharati Vidyapeeth University, Erandwane, Pune 411038, Maharashtra, INDIA.

Phone no: +91 02025437237

Email: kravi_73@rediffmail.com

DOI: 10.5530/ijpi.2021.1.15

\section{INTRODUCTION}

Carbon Nanotubes (CNTs) are carbon allotropes with a nanostructure having a length-to-diameter ratio of more than 1,000,000. These cylindrical carbon molecules have innovative characteristic features, making them possibly auxiliary in numerous nanotechnology applications. Produced from the graphene sheet they exhibit distinctive mechanical features like high durability and high multifarious moduli. ${ }^{1}$ Multi-functional nanoparticles have been of great importance in various biomedical applications, such as delivery systems, biosensors, bio imaging and tissue engineering. ${ }^{2}$ Among the numerous materials for nanoparticles, materials based on carbon have expanded biomedical building applications, including nano diamonds (NDs), carbon nanotubes, graphene, carbon nano fibers, fullerenes and nano horns ${ }^{3}$ Carbon nanotubes $(\mathrm{CN})$ have numerous structures, length differences, kind of helicity; number of layers and thickness. ${ }^{4}$ There are two main types of $\mathrm{CN}$ that can have high structural perfection, these are Singlewalled carbon nanotubes (SWCNT) consisting of a single graphite sheet seamlessly wrapped into a cylindrical tube and Multiwall carbon nanotubes (MWCNT) comprising of several graphenes wrapped together into a tube of cylindrical shape. ${ }^{5}$

The arc-discharge technique produces high-quality MWCNTs and SWCNTs. MWCNTs do not need a catalyst for growth, while SWCNTs can only be grown in the presence of a catalyst making MWCNT less toxic than that of SWCNT. Thus, MWCNT is generally the carrier of choice for delivery of drugs for most of the researchers. The MWCNT is composed of numerous rolled coatings of sheets of graphene with internal diameters as little as those of the SWCNTs that may be up to $10 \mathrm{~nm}$. MWCNTs have an interlayer separation of roughly $0.34 \mathrm{~nm}$, which is rather more noteworthy than graphite $(0.335 \mathrm{~nm}) 4$. However, MWCNT exhibit advantages over SWCNT, such as ease of mass production and low product cost per unit. ${ }^{6}$ Nano carriers can release the active drug directly into the cells and surmount the biological hurdles to offer categorical tissue targeting hence drug distribution at the target is possible. ${ }^{7}$ The solubility and compatibility of CNTs can be amended to a great extent by functionalization. Nanotubes provide the ideal and unique surrounding for a drug molecule until it reaches the desired site as well as avoids degradation and reaction with healthy cells. Nanotubes also provide a means of delivery without the need to include solvents. ${ }^{8}$ Use of SWCNTs and MWCNT's for the delivery of anti-cancer drugs is a major development in the nanotechnology. Management of cancer by conventional chemotherapeutic agents generally harms the healthy tissues. In such case, delivery of drug through SWCNTs and MWCNTs frameworks are a promising way for conveying anti-cancer drugs. ${ }^{9}$ Crossing the barrier of blood-brain is also an important application of these carriers that is utilized in different drug deliveries, as many drugs suffer from an inability to reach tumors (the blood-brain barrier prevents drugs from destroying brain tumors. ${ }^{10,11}$ MWCNT based systems help in improving the controlled release of BCS class 2 drugs and enhance the solubility of class 4 drugs. The effect of CNTs on the entrapment efficiency is very pronounced. The entrapment efficiency increases significantly as an increase in MWCNTs concentration which may be 
due to the interaction among the drug and MWCNTs. These carriers reduce the general drug leakage from the formulation with entrapping more amount of drug. ${ }^{12}$ Better drug loading is possible due to network like structure or high acceptance ratio as well as needle like structure shows better drug deposition. In general, electrical and mechanical features of MWCNT can alter when they are functionalized, because of the structural defects that arise by bond breakage of organic groups; throughout chemical processes. ${ }^{13}$ The functionalization of carbon nanotubes is based on confirmation of organic moiety linkages to their tubular structure. Through the functionalization of carbon nanotubes, it is possible to modulate their physico-chemical properties, increasing their ease of dispersion, manipulation and process ability among others. ${ }^{14}$ For the solubilization of carbon nanotubes, the attachment of relatively large functional groups to the nanotubes is required. ${ }^{15}$ In this work the $\mathrm{CNTs}$ were functionalized using organic groups such as carbonyl $(\mathrm{C}=\mathrm{O})$ and hydroxyl $(\mathrm{O}-\mathrm{H})$. The characterization of the transformed structures was done by FT-IR spectroscopy, mass spectroscopy and TEM technique. A dry-powder inhaler (DPI) is a device that conveys drug to the lungs in the form of dry powder. DPIs are generally utilized to treat respiratory problems, for example, asthma, bronchitis and respiratory viruses. ${ }^{16}$ The system is capable of continuously generating consistent concentrations $\left(3-12 \mathrm{mg} / \mathrm{m}^{3}\right)$ of an MWCNT like the DPI for extended periods (5h/day, 5 days/week, for up to 4 weeks). MWCNT was employed as an inhalation carrier involved in targeted delivery and selective controlled release to optimize the efficacy of the proposed drug and minimize the systemic as well as local toxicity. The concentration-dependent increase in the retention of pure MWCNT in the lung is associated with the severity of toxicity and after functionalization this toxicity may be reduced or might be non-toxic. Importantly, MWCNT fibers are also observed in the sub pleural area and diaphragm which is the reason behind toxicity. This indicates that inhaled MWCNT get deposited deep inside the respiratory tract and may be translocate into the pleural cavity, and that exposure to MWCNT induces pathological transmutations in the lung and chest cavity. ${ }^{17,18}$ In this research work, acute inhalation toxicity of pure and functionalized multi wall carbon nano tube was evaluated by performing different parameters like hemogram, liver function test, kidney function test, and histopathology study on Wistar albino rat. In past multi-walled carbon nanotubes have never been utilized as aerosol for inhalation purpose, and none research showed its toxicity or safety profile, ${ }^{19}$ also carbon nanotubes have not been analyzed as a carrier in DPI. Thus, there was a need to do such research and to determine its safety which is focused here.

\section{MATERIALS AND METHODS}

\section{Materials}

MWCNTs were purchased from Applied Science Innovations Pvt. Ltd., Pune, India. All the other chemicals used for synthesis were procured from Sigma Aldrich Chemicals Inc., Bangalore, India, and distilled water was prepared from de ionized water in the lab.

\section{Functionalization of multi-walled carbon nanotubes}

Functionalization of MWCNT was carried out by the initial basic treatment followed by treatment with hydrochloric acid. This generated functionalized MWCNTs covalently. By this method, the primary essential treatment with hydrogen peroxide and ammonium hydroxide created oxidized MWCNTs and later the treatment with $\mathrm{HCl}$ delivered carboxylated MWCNTs. $500 \mathrm{mg}$ of MWCNT was dispersed in $25 \mathrm{ml}$ of the mixture of $25 \%$ ammonium hydroxide and 30\% hydrogen peroxide $(\mathrm{V}: \mathrm{V}=1: 1)$ in a $100 \mathrm{ml}$ round bottom flask, furnished with a condenser and dispersion was heated to $80^{\circ} \mathrm{C}$ and kept for $5 \mathrm{~h}$. The residue thus obtained was then washed thoroughly with water until neutral $\mathrm{pH}$ was attained and dried overnight in vacuum at $40^{\circ} \mathrm{C} .{ }^{20}$ The treatment with hydrochloric acid produced carboxylated MWCNTs. In this method $500 \mathrm{mg}$ of MWNCTs was placed in round bottom flask and $200 \mathrm{ml}$ of $\mathrm{HCl}$ was added. The resultant reaction mixture was stirred using magnetic stirrer for $2 \mathrm{~h}$, then diluted, filtered and washed with ultrapure water dried in vacuum overnight. ${ }^{11}$

\section{Characterization of pure and functionalized multi- walled carbon nanotubes}

Infrared (IR) spectrum of pure and functionalized MWCNT was obtained on a JASCO, V-530 FTIR in anhydrous IR grade potassium bromide. The morphology was examined by Transmission electron microscopy (TEM). The mass spectrum was recorded employing electrospray ionization technique on G6460A Triple Quad LC/MS/MS System (Agilent Technologies) at Poona College of Pharmacy, Food Testing Laboratory, Pune, India.

\section{Acute inhalation toxicity study of multi-walled carbon nanotubes and functionalized multi-walled carbon nanotubes}

For the determination of MWCNT and FMWCNT toxicity study, the research experiment was performed on healthy Wistar albino rats weighing between $180 \mathrm{~g}$ and $250 \mathrm{~g}$ (National Institute of Biosciences, Pune, India). They were trained in cages with a 12:12 hr dark/light cycle and humidity $(44-55 \%)$ controlled environment and were provided free access to standard food and tap water. The studies were performed in accordance to Organization for Economic Cooperation and Development (OECD) and Animal Research: Reporting of in vivo Experiments (ARRIVE) guidelines. All studies were approved by the Institutional Animal Ethics Committee (IAEC) of Poona College of Pharmacy (1703/PO/C/13/CPCSEA) Pune, India bearing protocol number CPCSEA, PCP/PCT 08/2017-2018. The experiments were conducted under the provisions of approved protocol only. The animals were fasted but provided free access to water overnight before first day of dosing. Animals were divided into three groups for each dose i.e. lower and higher $(n=6)$ each consisting of 3 males and 3 females. First group (control group) received distilled water orally. Other groups should have been given lower to higher dose i.e. $0.5 \mathrm{mg} / \mathrm{kg}$, however according to OECD guideline there is no need to perform the study for lower dose, hence it was performed for higher dose $5 \mathrm{mg} / \mathrm{kg}$ nose-only exposure by fabricated apparatus. After the first dosing daily parameters were noted i.e. body weight, food intake, and water intake. After 28 days, blood sample withdrawn by retro-orbital puncture from the rats, was collected in EDTA tubes from each animal under anaesthesia and used to determine cellular count. ${ }^{21}$ Each sample was centrifuged at $500 \mathrm{rpm}$ for $10 \mathrm{~min}$ at $4^{\circ} \mathrm{C}$; the cells in the pellet were washed in $0.5 \mathrm{~mL}$ saline and total cells were counted using automated cell counter (KX -21, Sysmex, India). In order to perform differential analysis, aliquots of the cells were placed onto slides and stained with Field's stain to identify eosinophil's, lymphocytes, macrophages or neutrophils using standard morphologic determinants. At the end of the procedure and as per protocol, the animals were anaesthetized using $\mathrm{CO}_{2}$ and euthanasia was performed on randomly selected one animal of each group to analyse the macroscopic external features of the lung, kidney, and heart tissue. These tissues were carefully removed and fixed in $10 \%$ buffered formalin and embedded in paraffin. Histology sections ( $5 \mu \mathrm{m}$ thick) were stained with haematoxylin and examined under a light microscope.

\section{Hematological and biochemical parameter analysis}

The parameters observed, evaluated and interpreted for the toxicity study were as hematological parameters (including Red blood cells [RBC.], 
white blood cells [W.B.C], hemoglobin [Hb], Platelet, packed cell volume $[\mathrm{PCV}]$, mean corpuscular volume $[\mathrm{MCV}]$, corpuscular hemoglobin $[\mathrm{MCH}]$ and mean corpuscular hemoglobin concentration $[\mathrm{MCHC}]$ ), biochemical parameters (Gamma-glutamyltransferase [GGT], Total protein, albumin, globulin, serum glutamic-oxaloacetic transaminase [SGOT], serum glutamic-pyruvic transaminase [SGPT], alkaline phosphatase [ALP], Total Bilirubin, conjugated bilirubin, unconjugated bilirubin, creatinine, Blood urea nitrogen [BUN], sodium, potassium, urea, chloride and calcium.

\section{Histopathology}

Sacrificed the animals at end of study, organs were removed, weighed and finally processed for histopathology of kidney, heart, and lung. ${ }^{22,23}$

\section{RESULTS}

\section{Functionalization of Carbon Nanotubes}

Initial MWCNTs treated with ammonium hydroxide and hydrogen peroxide for oxidization of MWCNTs and again carboxylated by treatment with hydrochloric acid. $\mathrm{HCl}$ was used to produce covalently functionalized MWCNTs after successful functionalized characterize analytically.

\section{IR Spectroscopy}

The FTIR spectra of MWCNT and FMCNT revealed few common peaks at $2397.08 \mathrm{~cm}^{-1}$ and $2394.19 \mathrm{~cm}^{-1}$ respectively, however the FTIR spectrum of MWCNT showed peaks at $1691.27(\mathrm{C}=\mathrm{O})$ and $3287.07 \mathrm{~cm}^{-1}(-\mathrm{OH})$ confirming the attachment of $\mathrm{COOH}$ group after functionalization. The pure MWCNT had no obvious characteristic absorption peaks. IR spectra of pure and functionalized MWCNT obtained are as shown in Figure 1.

\section{Transmission electron microscopy}

The TEM image showed more bents with closed tips in functionalized MWCNT group as compared with the pure MWCNT group. TEM micrograph of pure MWCNT is Figure 2.

\section{Mass spectroscopy}

The results obtained from mass spectroscopy indicated the maintenance of the graphitic structure of MWCNTs after functionalization. Results for the pure MWCNT group showed the mass to charge ratio of 526.39

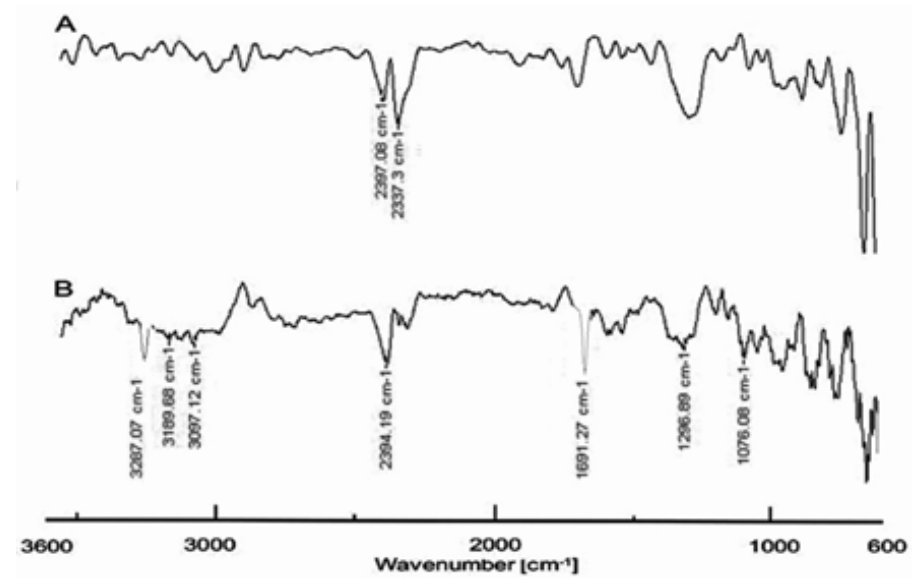

Figure 1: IR spectroscopy of pure MWCN (A) and functionalized MWCNT (B). $m / z$ as presented in Figure $3 \mathrm{~A}$, whereas for functionalized MWCNT group it was $942.49 \mathrm{~m} / \mathrm{z}$ as Figure $3 \mathrm{~B}$.

\section{Acute inhalation toxicity study of MWCNT and FMWCNT}

Daily parameters: Body weight (grams), water intake $(\mathrm{mL})$, and food intake (grams) were the daily parameters that were recorded, the results obtained showed that there was variation in body weight (grams), water intake $(\mathrm{mL})$, and food intake (grams) of both male and female Wistar rats after 28 days in pure MWCNT whereas functionalized MWCNT group and control group exhibited negligible difference.

Post sacrifices parameters: The results obtained for various parameters after 28 days study was as follows:

\section{Hemogram}

The values estimated for all hematological parameters of FMWCNT treated groups were comparable to that of control group as shown in Table 1. FMWCNT notably increased the Hb, WBC, platelets, PCV and $\mathrm{MCH}$ counts and lowered the MCV and MCHC counts compared to MWCNT indicating there were no alterations in any of the hematological parameters for control group and functionalized MWCNT group.

\section{Liver function test}

All the important liver function markers were quantified for all the three groups and the results obtained are Table 2. The results demonstrated that there was distinguished variation in all the parameters except the

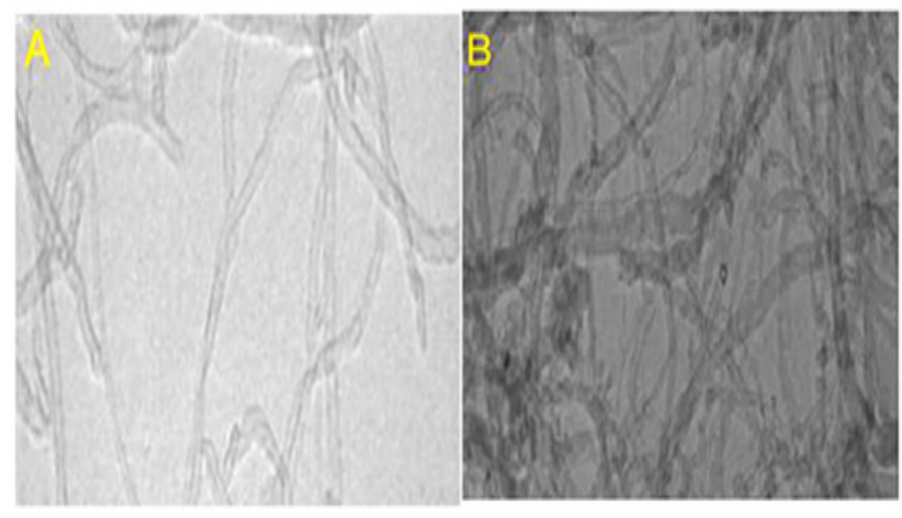

Figure 2: Morphology of pure MWCNT (TEM) (A) and functionalized MWCNT (TEM) (B).

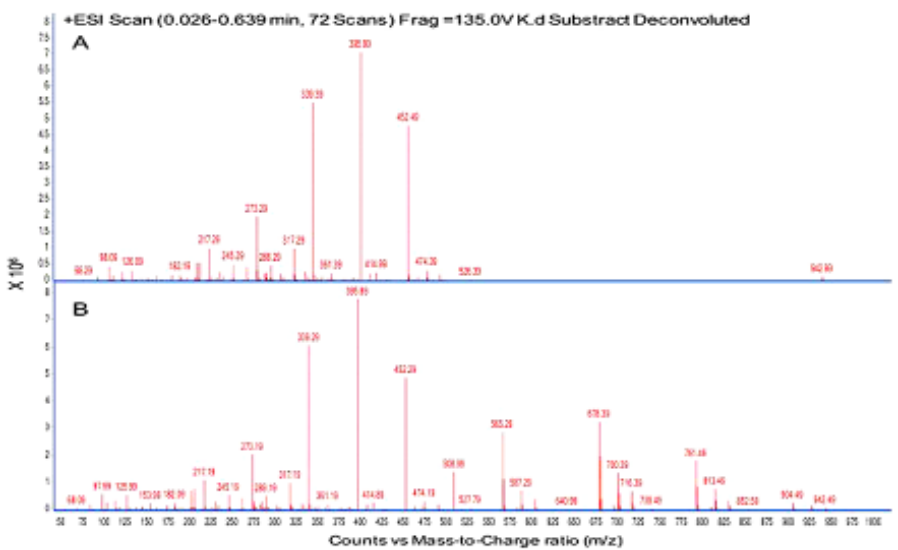

Figure 3: Mass spectroscopy of pure MWCNT (A) and functionalized MWCNT (B). 
GGT in pure MWCNT group compared to control and functionalized group.

\section{Kidney function test}

Kidney function test parameters were studied and results are depicted in Table 3. The values of creatinine, sodium, chloride, urea and BUN indicated alterations in MWCNT group compared to control and FMWCNT treated group, whereas levels of potassium were similar in all three groups.

Table 1: Haemogram study.

\begin{tabular}{cccc}
\hline Parameter & $\begin{array}{c}\text { Group 1 } \\
\text { Control group }\end{array}$ & $\begin{array}{c}\text { Group 2 } \\
\text { MWCNTs }\end{array}$ & $\begin{array}{c}\text { Group 3 } \\
\text { FMWCNTs }\end{array}$ \\
\hline $\mathrm{Hb}(\mathrm{g} / \mathrm{dl})$ & $13.10 \pm 0.29$ & $9.10 \pm 0.92 \#$ & $13.11 \pm 0.47 \mathrm{~ns}$ \\
$\mathrm{WBCs}(103 / \mathrm{mm} 3)$ & $9.50 \pm 0.15$ & $8.03 \pm 0.10 \#$ & $9.51 \pm * *$ \\
$\mathrm{RBCs}(103 / \mathrm{mm} 3)$ & $6.4 \pm 0.2$ & $6.4 \pm 0.47 \mathrm{~ns}$ & $6.4 \pm 0.15 \mathrm{~ns}$ \\
Platelets $(103 /$ & $480 \pm 2.31$ & $350 \pm 2.11 \#$ & $482 \pm 1.31 \mathrm{~ns}$ \\
$\mathrm{~mm} 3)$ & $39 \pm 1.3$ & $37 \pm 2.6 \#$ & $39 \pm 1.4 \mathrm{~ns}$ \\
$\mathrm{PCV}(\mathrm{mg} / \mathrm{dl})$ & $61 \pm 1.70$ & $63 \pm 0.79 \#$ & $60 \pm 0.94 \mathrm{~ns}$ \\
$\mathrm{MCV}(\mu \mathrm{m} 3)$ & $20 \pm 0.34$ & $17 \pm 0.31 \#$ & $20 \pm 0.26 \mathrm{~ns}$ \\
$\mathrm{MCH}(\mathrm{mmg})$ & $34 \pm 0.50$ & $37 \pm 0.23 \#$ & $35 \pm 1.10^{*}$ \\
\hline $\mathrm{MCHC}(\mathrm{mg} / \mathrm{dl})$ & & & \\
\hline
\end{tabular}

Data are expressed as mean $(n=6)$ and analyzed by one-way ANOVA followed by Dunnetts test for each parameter of FMWCNTs group 3 separately. ${ }^{*} P<0.05,{ }^{*} P<0.01,{ }^{* *} P<0.001$ and FMWCNTs group 2 separately $\# P<0.05, \# \# P<0.01, \# \# \#<0.001$, as compared to control group and. ns non significant as compared to Normal group

\section{Table 2: Liver function test.}

\begin{tabular}{|c|c|c|c|}
\hline Parameter & $\begin{array}{c}\text { Group } 1 \\
\text { Control } \\
\text { group }\end{array}$ & $\begin{array}{l}\text { Group } 2 \\
\text { MWCNTs }\end{array}$ & $\begin{array}{c}\text { Group } 3 \\
\text { FMWCNTs }\end{array}$ \\
\hline Total Protein (g/l) & $8.55 \pm 1.06$ & $7.85 \pm 0.49$ \#\# & $8.35 \pm 0.91^{* * *}$ \\
\hline Albumin (g/dl) & $4 \pm 0.02$ & $6 \pm 0.01 \#$ & $4.85 \pm 0.02^{* * *}$ \\
\hline Globulin (g/dl) & $6.85 \pm 0.07$ & $6.4 \pm 0.28 \#$ & $6.85 \pm 0.35^{* * *}$ \\
\hline $\begin{array}{l}\text { Alkaline phosphotase } \\
\qquad(\mathrm{u} / \mathrm{l})\end{array}$ & $168 \pm 6.79$ & $158 \pm 21.21 \# \# \#$ & $162 \pm 14.14^{* *}$ \\
\hline SGPT (u/l) & $95 \pm 8.48$ & 93.5 $99.19 \# \# \#$ & $92.5 \pm 10.60^{* *}$ \\
\hline SGOT (u/l) & $252.5 \pm 24.74$ & $249.5 \pm 33.23 \# \# \#$ & $247 \pm 22.62$ ** \\
\hline Total Bilirubin (mg/dl) & $0.2 \pm 0.02$ & $0.05 \pm 0.01 \#$ & $\underset{* * *}{0.185 \pm 0.02}$ \\
\hline $\begin{array}{l}\text { Conjugated Bilirubin } \\
\qquad(\mathrm{mg} / \mathrm{dl})\end{array}$ & $0.0375 \pm 0.01$ & $0.0255 \pm 0.00 \#$ & $0.039 \pm 0.00^{*}$ \\
\hline $\begin{array}{l}\text { Unconjugated bilirubin } \\
\qquad(\mathrm{mg} / \mathrm{dl})\end{array}$ & $0.165 \pm 0.03$ & $0.095 \pm 0.03 \#$ & $0.155 \pm 0.00^{*}$ \\
\hline GGTP $(\mathrm{u} / \mathrm{l})$ & $8 \pm 1.4$ & $8 \pm 2.8 \mathrm{~ns}$ & $8.65 \pm 1.2^{* * *}$ \\
\hline
\end{tabular}

Data are expressed as mean $(n=6)$ and analyzed by one-way ANOVA followed by Dunnetts test for each parameter of FMWCNTs group 3 separately. ${ }^{*} P<0.05$, ${ }^{*} P<0.01,{ }^{* *} P<0.001$ and FMWCNTs group 2 separately $\# P<0.05$, \#\#P< 0.01 , \#\#P $<0.001$, as compared to control group and. ns non significant as compared to Normal group

\section{Histopathology}

Histopathological investigation of sections of rat lung, kidney, and heart taken from the MWCNT-administered group displayed histological deterioration in the form of inflammation, blood clots, necrosis etc. Whereas there were no variations in tissue sections of FMWCNT when compared with control group as shown in Figures 4-6.

\section{DISCUSSION}

Functionalization of MWCNT to FMWCNT was undertaken with the purpose of reducing the toxicity. ${ }^{24}$ It was performed via initial basic treatment with ammonium hydroxide and hydrogen peroxide produced oxidized MWCNTs then simple acidic treatment procedure
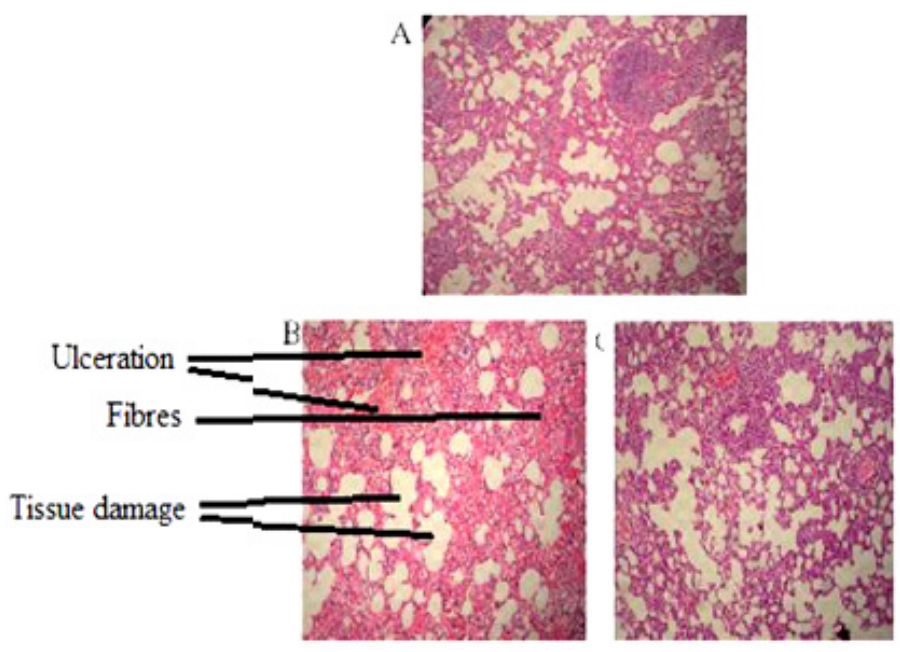

Figure 4: Histopathology of lung (A: control group, B: pure MWCNT, C: functionalized MWCNT, TD: tissue damage, U: ulceration).

Table 3: Kidney function test.

\begin{tabular}{cccc}
\hline Parameter & $\begin{array}{c}\text { Group 1 } \\
\text { Control } \\
\text { group }\end{array}$ & $\begin{array}{c}\text { Group 2 } \\
\text { MWCNTs }\end{array}$ & $\begin{array}{c}\text { Group 3 } \\
\text { FMWCNTs }\end{array}$ \\
\hline Creatinine (mg/dl) & $0.83 \pm 0.08$ & $1.29 \pm 0.04 \#$ & $0.82 \pm 0.05^{* * *}$ \\
Sodium (m mol/lit) & $124 \pm 31.11$ & $118.5 \pm 23.33 \# \#$ & $\begin{array}{c}125.5 \pm 30.40 \\
\mathrm{~ns}\end{array}$ \\
Potassium (m mol/lit) & $4.35 \pm 0.35$ & $4.3 \pm 0.28 \mathrm{~ns}$ & $4.5 \pm 0.28 \mathrm{~ns}$ \\
Chloride (m mol/lit) & $99 \pm 0.00$ & $96.5 \pm 2.12 \#$ & $100 \pm 0.00^{* * *}$ \\
Urea (mg/dl) & $46.5 \pm 4.94$ & $50.5 \pm 6.36 \# \#$ & $45 \pm 5.65 \mathrm{~ns}$ \\
BUN (blood urea & $16 \pm 4.24$ & $22.5 \pm 0.70 \#$ & $16.5 \pm 3.53 \mathrm{~ns}$ \\
nitrogen) (mg/dl) & & & \\
BicarbonatemEq/1 & $22.5 \pm 2.12$ & $22 \pm 1.41 \# \# \#$ & $22.5 \pm 2.12 \mathrm{~ns}$ \\
Calcium (mmol/L) & $9.65 \pm 0.49$ & $9.45 \pm 0.49 \#$ & $9.65 \pm 1.13 \mathrm{~ns}$ \\
Phosphorus (mg/dL) & $6.8 \pm 0.98$ & $6.75 \pm 1.27 \mathrm{~ns}$ & $6.78 \pm 1.20 \mathrm{~ns}$ \\
Uric acid (mg/dL) & $0.98 \pm 0.01$ & $2.45 \pm 0.07 \#$ & $1 \pm 0.00^{* * *}$ \\
\hline
\end{tabular}

Data are expressed as mean $(n=6)$ and analyzed by one-way ANOVA followed by Dunnetts test for each parameter of FMWCNTs group 3 separately. ${ }^{*}<0.05$, ${ }^{* *} P<0.01,{ }^{* *} P<0.001$ and FMWCNTs group 2 separately $\# P<0.05$, \#\#P< 0.01 , \#\#P $<0.001$, as compared to control group and. Ns non significant as compared to Normal group. 

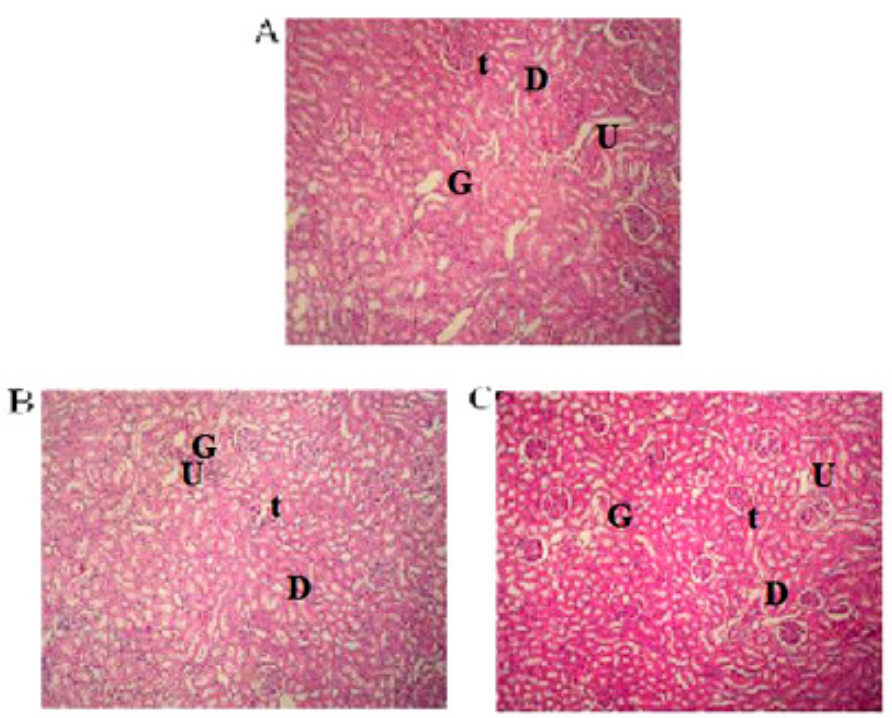

Figure 5: Histopathology of kidney (A: control group, B: pure MWCNT, C: functionalized MWCNT) $\mathrm{G}$ - glomerulus, $\mathrm{U}$ - urinary space, $\mathrm{t}$ - proximal convoluted tubule, $\mathrm{D}$ - distal convoluted tubule


Figure 6: Histopathology of heart (A: control group, B: pure MWCNT, C: functionalized.

and attachment of carboxylic functional group on the pure MWCNT was confirmed from the presence of characteristic peaks of $\mathrm{C}=\mathrm{O}$ and $\mathrm{O}-\mathrm{H}$ seen in IR spectrum of FMWCNT and absence of these peaks in the spectra of pure MWCNT. On account of functionalization several functional groups were attached which were observed in the form of bents in the TEM image. ${ }^{25}$ Notably pure MWCNTs were seen to have closed tips; it was found that fewer bents were present inside the structure, even after bent washing with water, thus confirming functionalization. From the increase in mass to charge ratio noted in mass spectrum of FMWCNT compared to pure MWCNT it was inferred that attachment of carboxylic functional group increased the molecular weight. The toxicity studies included quantification of various parameters like hematological, liver function markers, kidney function markers and histopathology. Significant diminution in levels of $\mathrm{Hb}, \mathrm{WBC}$, platelets, $\mathrm{PCV}$ and $\mathrm{MCH}$ counts and elevated levels of MCV and MCHC are consequences of underlying toxicity and were prominent in MWCNT treated group. ${ }^{26-28}$ In the present study, treatment with FMWCNT could successfully restore the hematological parameters comparable to the control group, signifying its ameliorating effect. Estimation of liver function markers demonstrated that there was distinguished variation in albumin and total bilirubin count of the pure MWCNT group compared with control and functionalized group. ${ }^{29}$ This can be attributed to the fact that when liver is in inflamed condition, it fails to process bilirubin and hence its levels rise in blood. Likewise elevated levels of albumin are associated with exposure to toxicants or may be due to dehydration and were observed in MWCNT treated group. ${ }^{30-32}$ Albumin levels in FMWCNT treated group were however similar to control group suggesting that FMWCNT does not cause any toxic alterations. The kidney function can be measured by estimating concentration of electrolytes sodium, potassium, chloride etc. and concentration of metabolites like urea and creatinine. The normal ranges of these parameters are confirmation of healthy renal functioning. In our study these levels were found to be disturbed in case of MWCNT- administered group. However, FMWCNT-administered group did not show variations in levels of creatinine, chloride, urea and BUN (similar to control group) which further ascertained the safety of FMWCNT. In histological examination of MWCNT treated rat lungs, signs of inflammation like ulceration, blood clots, tissue damage, inflammatory infiltrate and dense eosinophils were evident Figure 4. Images of control and FMWCNT treated rat lungs indicated intact cyto-architecture without any abnormality. (Figure 5) represents histopathology of the kidney, where exposures of MWCNT in rats produced significant tubular necrosis which was characterized by death of tubular epithelial cells of the kidney and interstitial nephritis. ${ }^{33}$ Which is swelling in between the kidney tubules at a dose of $5 \mathrm{mg}$ / $\mathrm{kg}$ at 28 days in pure MWCNT whereas no variation was observed in functionalized MWCNT in comparison with control group. From results of histopathology of heart shown in Figure 6. It can be stated that pure and functionalized MWCNT showed no significant changes with $5 \mathrm{mg} /$ $\mathrm{kg}$ dose in comparison with control group. Thus, no signs of toxicity were observed in FMWCNT-treated rat kidney and heart images, confirming its safety in vivo MWCNT in comparison with control group..$^{24,34}$ The current research described the results of in vivo toxicity studies conducted on functionalized (FMWCNT) and pure multi-walled carbon nanotubes (MWCNT). The justification for these particular tests rests on the criteria: (i.e., Daily parameters, Histopathology, Hb, WBC, $\mathrm{RBC}$, platelets, $\mathrm{PCV}, \mathrm{MCV}, \mathrm{MCH}$, ad $\mathrm{MCHC}$ ), liver function test and kidney function test in Table 1-3. And the results of these acute studies that did not show any significant changes also not demonstrated hazard potential in rats following acute exposures to the functionalized multiwalled carbon nanotubes (MWCNT) and no significant toxicity was observed. This may be accredited to the fact that acidic treatment during functionalization removes all impurities. During functionalization addition of oxygen containing group and removal of carbon dioxide group occurs. This may be the reason that FMWCNT is non-toxic.

\section{CONCLUSION}

Functionalization of MWCNT was performed successfully to assess material inhalation toxicity taking into consideration various parameters known to quantify toxicity. It was observed that the functionalization of MWCNT reduced material toxicity. Additionally, it can be concluded that MWCNT after functionalization is safe advanced material carrier and can act as potential drug carrier in inhalation drug delivery systems such as dry powder inhalers. Briefly, all these consequences open a new door for advanced carbon material in pulmonary drug delivery.

\section{ACKNOWLEDGEMENT}

Authors would like to thank to Dhande Pathlab Diagnostic Pvt. Ltd. Pune for their kind support during hematological and all other lab studies. 


\section{CONFLICT OF INTEREST}

The authors declare that there is no conflict of interest.

\section{ABBREVIATIONS}

FMWCNT: Functionalization of mult-walled carbon numbers; CNTs: Carbon Nanotubes; SWCNT: Single walled carbon nanotubes; IAEC: Institutional Animal Ethics Committee; ARRIVE: Animal Research: Reporting in vivo Experiments.

\section{REFERENCES}

1. Varshney K. Carbon nanotubes: A review on synthesis, properties and applications. Int J Eng Res Gen Sci. 2014;2(4): 660-677

2. Zhang Q, Mochalin VN, Neitzel I, Knoke IY, Han J, Klug CA, et al. Fluorescent PLLA nanodiamond composites for bone tissue engineering. Biomaterials. 2011;32(1):87-94.

3. Zhang BT, Zheng XL, Lin HFJM. Application of carbon-based nanomaterials in sample preparation: A review. Anal Chim Acta. 2013;784:1-17.

4. Obitayo W, Liu T. A review: Carbon nanotube-based piezoresistive strain sensors. Mater Sci Eng. 2012;43(3):61-102.

5. Khare R. Carbon nanotube-based composites: A review. Mater Charact Eng. 2005;4(1):31.

6. Burke A, Ding X, Singh R, Kraft RA, Levi-Polyachenko N, Rylander MN, et al. Long-term survival following a single treatment of kidney tumors with multi walled carbon nanotubes and near-infrared radiation. Proc Natl Acad Sci. 2009;106(31):12897-902

7. Cirillo G, Hampel S, Spizzirri UG, Parisi Ol, Picci N, lemma F, et al. Carbon nanotubes hybrid hydrogels in drug delivery: A perspective review. BioMed Res Int. 2014:825017.

8. HilderTA, Hill JM. Modeling the loading and unloading of drugs into nanotubes. Small. 2009;5(3):300-8.

9. Dinesh KB, Krishna KK, Bhatt AR, Paul D, Cherian J, John A, et al. Singlewalled and multi-walled carbon nanotubes based drug delivery system: Cancer therapy: A review. Indian J Cancer. 2015;52(3):262

10. Beg S, Rizwan M, Sheikh AM, Hasnain MS, Anwer K, Kohli K, et al. Advancement in carbon nanotubes: Basics, biomedical applications and toxicity. J Pharm Pharmacol. 2011;6(32):141-63.

11. Ghoshal S, Kushwaha SKS, Srivastava M, Tiwari P. Drug loading and release from functionalized multiwalled carbon nanotubes loaded with 6-mercaptopurine using incipient wetness impregnation method. Am J Adv Drug Deliv. 2014;2(2):213-23.

12. Garala K, Patel J, Patel A, Dharamsi A. Enhanced encapsulation of metoprolol tartrate with carbon nanotubes as adsorbent. Appl Nanosci. 2011;1(4):219-30.

13. Ngo CL, Le QT, Ngo TT, Nguyen DN, Vu MT. Surface modification and functionalization of carbon nanotube with some organic compounds. Nanosci Nanotechnol. 2013;4(3):035017.

14. Ballesteros B, Tobias G, Shao L, Pellicer E, Nogués, J, Mendoza E, et al. Steam Purification for the Removal of Graphitic Shells Coating Catalytic Particles and the Shortening of Single-Walled Carbon Nanotubes. Small. 2008;4(9):1501-6.

15. SunYP, Fu K, Lin Y, Huang W. Functionalized carbon nanotubes: Properties and applications. Acc Chem Res. 2002;35(12):1096-104.
16. Endo M, Strano MS, Ajayan PM. Potential applications of carbon nanotubes. Carbon nanotubes. 2007; 13:62.

17. Chen BT, Schwegler-Berry D, McKinney W, Stone S, Cumpston JL, Friend S, et al. Multi-walled carbon nanotubes: Sampling criteria and aerosol characterization. Inhal Toxicol. 2012;24(12):798-820.

18. Kasai $T$, Umeda $Y$, Ohnishi M, Mine $T$, Kondo $H$, Takeuchi $T$, et al. Lung carcinogenicity of inhaled multi-walled carbon nanotube in rats. Part Fibretoxicol. 2015;13(1):153.

19. Takafumi S, Katsuhide F. A review of pulmonary toxicity studies of nanocellulose. Inhal Toxicity. 2020;1-8.

20. Kim JK, Jo MS, Kim Y, Kim TG, Shin JH, Kim BW, et al. 28-Day inhalation toxicity study with evaluation of lung deposition and retention of tangled multi-walled carbon nanotubes. Nanotoxicology. 2020;14(2):250-62.

21. Parasuraman $S$, Zhen KM, Raveendran R. Retro-orbital sample collection in Rats-a video article. Pharmacol, Toxicol Biomed Rep. 2015;1(2):37-40.

22. Mali AJ, Bothiraja C, Purohit RN, Pawar AP. In vitro and in vivo performance of novel spray dried andrographolide loaded scleroglucan based formulation for dry powder inhaler. Curr Drugdeliv. 2017;14(7):968-80.

23. Lehman JH, Terrones M, Mansfield E, Hurst KE, Meunier V. Evaluating the characteristics of multiwall carbon nanotubes. Carbon. 2011;49(8):2581-602.

24. Ivana F, Elisabetta A, Elena G, Federico C, Massimiliano C, Domenica S, et al. Thickness of multiwalled carbon nanotubes affects their lung toxicity. Chem Res Toxicol. 2012;25(1):74-82.

25. Yasuomorimoto $M$, Horie $N$, Kobayashi N, Shinohara MS. Inhalation toxicity assessment of carbon-based nanoparticles. Accounts Chem Res. 2013;46(3):770-81.

26. Zare-Zardini $H$, Amiri A, Shanbedi $M$. In vitro and in vivo study of hazardous effects of Ag nanoparticles and Arginine-treated multi walled carbon nanotubes on blood cells: Application in hemodialysis membranes. J Biomed Mater Res A. 2015;103(9):2959-65.

27. Bhilare NV, Dhaneshwar SS. Synthesis and evaluation of morpholinoethyl ester conjugate of $\mathrm{N}$-acetylcysteine in ovalbumin induced airway hyperresponsiveness in Sprague Dawley rats Letters. Drug Design and Discovery. 2017;14(2):209-15.

28. Tavabe KR, Yavar M, Kabir S, Akbary P, Aminikhoei Z. Toxicity effects of multiwalled carbon nanotubes (MWCNTs) nanomaterial on the common carp (Cyprinuscarpio L 1758) in laboratory conditions Comparative Biochemistry and Physiology Part C. Toxicol Pharmacol. 2020;108832.

29. Emerce E, Ghosh M, Öner D, Radu-Corneliu D, Jeroen D, Vanoirbeek J, et al. Carbon Nanotube- and Asbestos-Induced DNA and RNA Methylation Changes in Bronchial Epithelial Cells. Chem Res Toxicol. 2019;32(5):850-60.

30. Bhilare NV, Dhaneshwar SS, Mahadik KR, Dasgupta A. Co-drug of isoniazid and sulfur containing antioxidant for attenuation of hepatotoxicity and treatment of tuberculosis. Drug Che Toxicol. 2020;1:11.

31. Awotunde OS, Adewoye SO, Hawumba J. Subacute toxicity study of aqueous root extract of Terminaliaschimperiana in male Wistar rats. Toxicol Rep. 2019;6:825-32.

32. Bhilare NV, Dhaneshwar SS, Mahadik KR Amelioration of hepatotoxicity by biocleavableaminothiol chimeras of isoniazid: Design, synthesis, kinetics and pharmacological evaluation. World J Hepatol. 2018;10(7):496-508.

33. Jain S, Thakare VS, Das M, Godugu C, Jain AK, Mathur R, et al. Toxicity of multiwalled carbon nanotubes with end defects critically depends on their functionalization. Density Chem Res Toxicol. 2011;24(11):2028-39.

34. Liu Y, Zhao Y, Sun B, Chen C. Understanding the Toxicity of Carbon Nanotubes. Acc Chem Res. 2013;46(3):702-13.

Article History: Submission Date : 16-12-2020; Revised Date : 17-01-2021; Acceptance Date : 27-02-2021

Cite this article: Dhekale K, Patil S, Kamble R. Advanced Development of Carboxylic Acid Functionalized Multiwall Carbon Nanotubes as safe Inhalation Drug Carrier. Int. J. Pharm. Investigation. 2021;11(1):82-7. 